\title{
An Atomic Level Study of Local Strain Fields on Multiple Low-index Ceria $\left(\mathrm{CeO}_{2}\right)$ Nanoparticle Surfaces
}

\author{
Piyush Haluai, Ethan Lawrence, Barnaby Levin and Peter Crozier
}

Arizona State University, Tempe, Arizona, United States

The surface strain of oxide nanoparticles plays a crucial role in tuning functional materials' properties [1]. For example, surface strain can regulate and control surface diffusion processes and can change the ability of the surface atoms to bond with adsorbates and thus modify the reactivity of a material [2]. In reducible oxides such as $\mathrm{CeO}_{2}$, the degree of strain can be tuned by different means such as particle size, shape, non-stoichiometry, ambient environment (e.g. oxygen atmosphere, vacuum), structural deformations (e.g. oxygen vacancies) etc. [3]. A critical step in understanding the properties of nanoparticle surfaces is to measure the surface strain on different crystal facets and in the vicinity of surface defects in different ambient environments. We have developed a method to determine the surface strain in oxide nanoparticles with atomic resolution using high resolution transmission electron microscopy (HRTEM). In a typical TEM image, the signal from the heavier Ce atomic columns is much stronger than the signal from the lighter oxygen columns. Consequently, more precise measurements can be made on the cation sublattice in order to map the strain on or near the nanoparticle surface.

$\mathrm{CeO}_{2}$ nanoparticles were synthesized by the hydrothermal method [4] and imaged using negative Cs imaging in a FEI Titan AC-ETEM with a single-electron-detection K2 camera operated in the counting mode. The K2 camera allowed high quality electron imaging to be performed. (111), (110), and (100) $\mathrm{CeO}_{2}$ nanoparticle surfaces were imaged in a [110] projection at $5000 \mathrm{e}^{-} \AA^{-2} \mathrm{~s}^{-1}$ with $\mathrm{Ce}$ and $\mathrm{O}$ atomic columns visible at the surface [5]. Custom written MATLAB codes are used to identify and determine the positions of atomic columns by fitting a two dimensional (2D) elliptical Gaussian to each atomic column [6]. This technique allows picoscale precision. Atomic resolution strain maps were created to visualize cation sublattice deformations at different locations in the nanoparticle. The strain is calculated based on a relative change of cation sublattice at the surface of the nanoparticle to the bulk. In vacuum environment inside the microscope, the bulk of the nanoparticle is relatively strain free, but the surfaces show varying degrees of compressive and tensile strain along different crystallographic directions of the nanoparticle. The surface strain field varies in a complicated fashion with the highest tensile and compressive strains along different directions occurring at different points on the surface. The highest degree of strain is associated with defects such as step sites. These local strain fields on $\mathrm{CeO}_{2}$ nanoparticle surfaces are correlated with local activity for oxygen vacancy creation and annihilation. We are currently measuring the strain maps in oxygen environment inside the microscope and the findings will be discussed during the time of presentation [7].

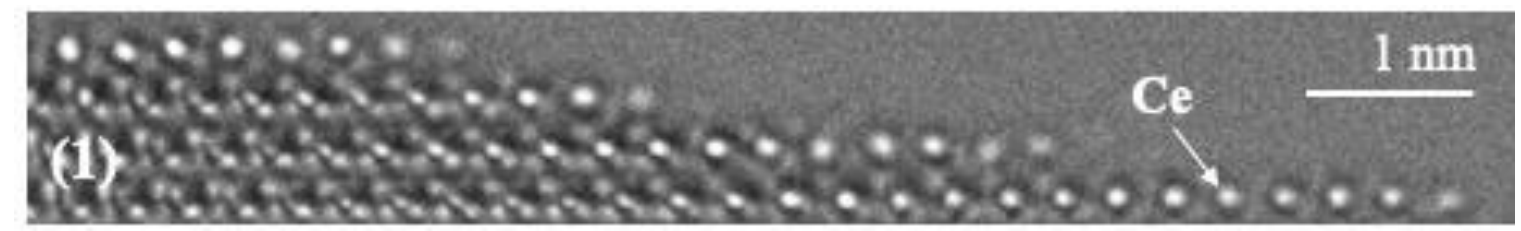

Figure 1. Ceria nanoparticle imaged in [110] projection. The Ce atomic column positions appear as bright dots due to negative Cs imaging (marked by white arrow). 


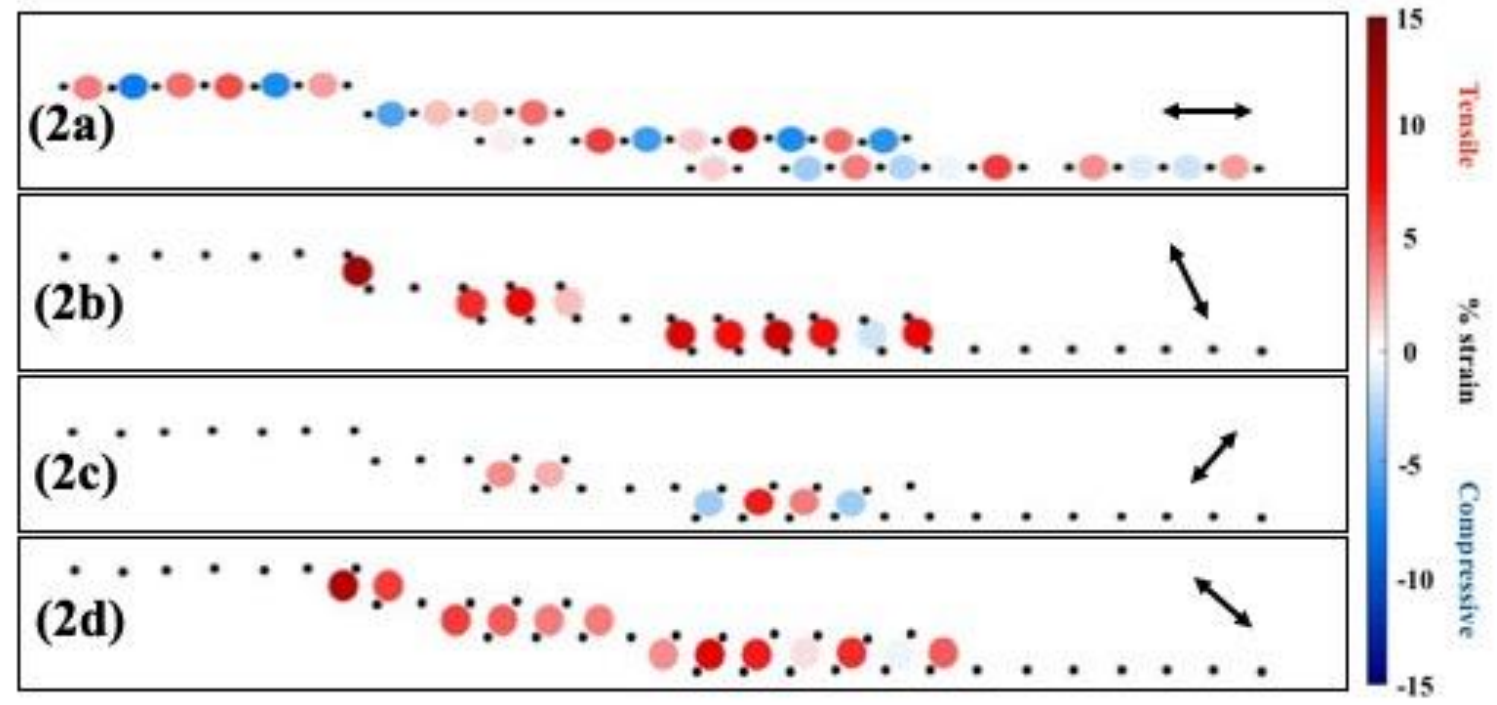

Figure 2. 2a, 2b, 2c, and $2 \mathrm{~d}$ show the strain fields (corresponding to Figure 1) along different crystallographic directions marked by the double-sided arrows. The black dots represent the cation sublattice (Ce atomic columns) The scale bar corresponding to \% strain is shown in the side.

References

1. Madsen, Jacob, et al.; Advanced structural and chemical imaging 1 (2017): 14.

2. Mavrikakis, M, et al.; Physical Review Letters 13 (1998): 2819.

3. Gopal, Chirranjeevi Balaji, et al.; Nature communications 8 (2017): 15360.

4. Yang, Zhiqiang, et al.; Nanotechnology 18 (2007): 185606.

5. Lawrence, E. L., et al.; Microscopy and Microanalysis 24 S1 (2018), p, 54-55

6. Levin, B.D.A. et al.; (2019) arXiv:1909.07477

7. We gratefully acknowledge support of NSF grant DMR-1308085, DOE (DE-SC0004954), the use of ASU's John M. Cowley Center for High Resolution Electron Microscopy and use of the K2 IS camera courtesy of Gatan. 